\title{
Impact of Dynamic Computed Tomographic Angiography on Endograft Sizing for Endovascular Aneurysm Repair
}

\author{
Jillis A. Pol, MD"; Maarten Truijers, MD, PhD'; J. Adam van der Vliet, MD, PhD; \\ Mark F. Fillinger, MD2; Steven P. Marra, PhD3; W. Klaas Jan Renema, PhD4; \\ Luuk J. Oostveen, BSc ${ }^{4}$; Leo J. Schultze Kool, MD, PhD4; and \\ Jan D. Blankensteijn, MD, PhD ${ }^{1}$
}

1Department of Surgery, Division of Vascular and Transplant Surgery, and ${ }^{4}$ Department of Radiology, Radboud University Nijmegen Medical Center, Nijmegen, The Netherlands. ${ }^{2}$ Department of Vascular Surgery, Dartmouth-Hitchcock Medical Center, Lebanon, New Hampshire, USA. ${ }^{3} \mathrm{M} 2 \mathrm{~S}$ Inc, Lebanon, New Hampshire, USA.

Purpose: To quantify dynamic changes in aortoiliac dimensions using dynamic electrocardiographically (ECG)-gated computed tomographic angiography (CTA) and to investigate any potential impact on preoperative endograft sizing in relation to observer variability.

Methods: Dynamic ECG-gated CTA was performed in 18 patients with abdominal aortic aneurysms. Postprocessing resulted in 11 datasets per patient: 1 static CTA and 10 dynamic CTA series. Vessel diameter, length, and angulation were measured for all phases of the cardiac cycle. The differences between diastolic and systolic aneurysm dimensions were analyzed for significance using paired $t$ tests. To assess intraobserver variability, 20 randomly selected datasets were analyzed twice. Intraobserver repeatability coefficients (RC) were calculated using Bland-Altman analysis.

Results: Mean aortic diameter at the proximal neck was $21.4 \pm 3.0 \mathrm{~mm}$ at diastole and $23.2 \pm 2.9 \mathrm{~mm}$ at systole, a mean increase of $1.8 \pm 0.4 \mathrm{~mm}(8.5 \%, p<0.01)$. The $\mathrm{RC}$ for the aortic diameter at the level of the proximal aneurysm neck was $1.9 \mathrm{~mm}$ (8.9\%). At the distal sealing zones, the mean increase in diameter was $1.7 \pm 0.3 \mathrm{~mm}(14.1 \%, \mathrm{p}<0.01)$ for the right and $1.8 \pm 0.5 \mathrm{~mm}(14.2 \%, \mathrm{p}<0.01)$ for the left common iliac artery (CIA). At both distal sealing zones, the mean increase in $\mathrm{CIA}$ diameter exceeded the $\mathrm{RC}(10.0 \%$ for the right $\mathrm{CIA}$ and $12.6 \%$ for the left $\mathrm{CIA}$ ).

Conclusion: The observed changes in aneurysm dimension during the cardiac cycle are small and in the range of intraobserver variability, so dynamic changes in proximal aneurysm neck diameter and aneurysm length likely have little impact on preoperative endograft selection. However, changes in diameter at the distal sealing zones may be relevant to sizing, so distal oversizing of up to $20 \%$ should be considered to prevent distal type I endoleak.

J Endovasc Ther. 2009;16:546-551

Key words: abdominal aortic aneurysm, dynamic computed tomographic angiography, endovascular aneurysm repair, electrocardiography, stent-graft

Steven Marra is an employee of M2S Inc, Lebanon, New Hampshire, USA. The other authors have no commercial, proprietary, or financial interest in any products or companies described in this article.

Address for correspondence and reprints: Jillis A. Pol, MD, 690 Department of Surgery, Division of Vascular and Transplant Surgery, Radboud University Nijmegen Medical Center, POB 9101, 6500 HB Nijmegen, The Netherlands. E-mail: J.Pol@chir.umcn.nl 
The success of endovascular aneurysm repair (EVAR) depends on an accurate preoperative assessment of aortoiliac morphology. ${ }^{1-3}$ Since the introduction of EVAR, several imaging modalities have been proposed for the preoperative workup of patients with abdominal aortic aneurysm (AAA)., ${ }^{4,5}$ Computed tomographic angiography (CTA) is the only modality that provides all necessary data for a complete preoperative assessment of AAA anatomy. ${ }^{6-8}$

The development of faster multislice CT scanners and innovative image postprocessing

\section{See commentary page 552}

algorithms has further expanded the capabilities of CTA to include, among other things, dynamic imaging. In contrast to conventional or static CTA, dynamic CTA allows data and image reconstruction for any given phase of the cardiac cycle, which can provide valuable information on aortic pulsatility and dynamic changes in aneurysm morphology. ${ }^{9-12}$ The aim of the present study was to quantify dynamic changes in aortoiliac dimensions and investigate any potential impact on preoperative endograft sizing in relation to observer variability of dynamic CTA measurements.

\section{METHODS}

\section{Data Acquisition and Image Postprocessing}

Dynamic CTA was performed in 18 AAA patients referred for diagnostic imaging; the aneurysm diameter ranged from 3.8 to $7.0 \mathrm{~cm}$. The study protocol was approved by the local ethics committee, and written informed consent was obtained in all participants.

Patients were scanned using a 64-row multidetector CT scanner (Somatom Sensation; Siemens, Erlangen, Germany) with simultaneous ECG registration. Data acquisition started after reaching a predefined contrast enhancement threshold (bolus triggering) seconds after intravenous contrast administration (Xenetix 350; Guerbet, Paris, France). Radiation dose and scanning parameters were similar to conventional CTA and included a tube voltage of $120 \mathrm{kV}, 120 \mathrm{mAs}$, and a pitch of 0.34 .

Data postprocessing resulted in 11 datasets per patient, 1 conventional or static CTA and

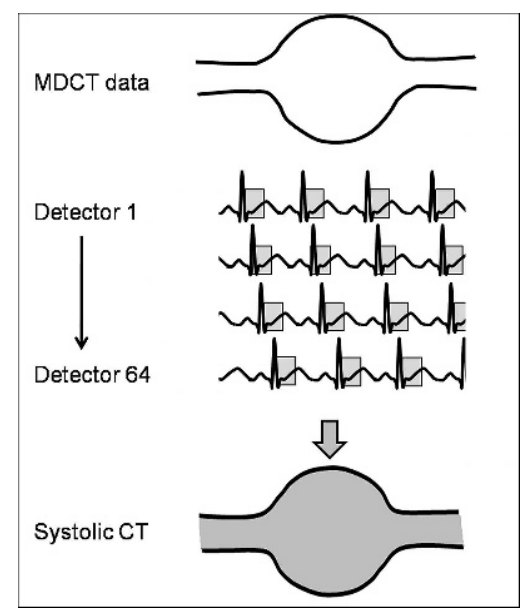

Figure $1 \diamond$ Dynamic retrospective ECG-gated CTA is based upon conventional multislice CT (MSCT), which allows simultaneous acquisition of multiple slices during one gantry rotation to produce images with high spatial and temporal resolution (top). Using simultaneous ECG registration and an algorithm for retrospective gating, MSCT enables dynamic CTA. As the gantry starts rotating, the first detector acquires data at the proximal aneurysm neck in systole (top ECG row). Because of the small pitch (0.34), the last of the 64 detectors acquires data at the proximal aneurysm neck while the cardiac cycle moves to diastole (bottom ECG row). Combining data from all detectors during systole results in a systolic ECG-gated dataset (bottom row).

10 dynamic CTA series. Each of the dynamic series represented a different phase of the cardiac cycle (Fig. 1). CTA header information was removed, and all 198 scans were analyzed in random order. One-millimeter-thick cross-sectional images (perpendicular to the central luminal line) and 3-dimensional (3D) aneurysm models (Fig. 2) were reconstructed (Preview 2.9.0; M2S Inc, Lebanon, NH, USA) for the purpose of diameter, length, and angulation measurement. ${ }^{8,13-17}$ These 3D models were created using automated segmentation on each axial slice where lumen, thrombus, and calcifications were distinguished by color coding; each segmentation was reviewed manually and adjusted for small irregularities. Subsequently, all axial images are transformed to a 3D model.

\section{Aortic Dimensions}

Eleven different indices of aortoiliac morphology (Fig. 3) were quantified. For each 


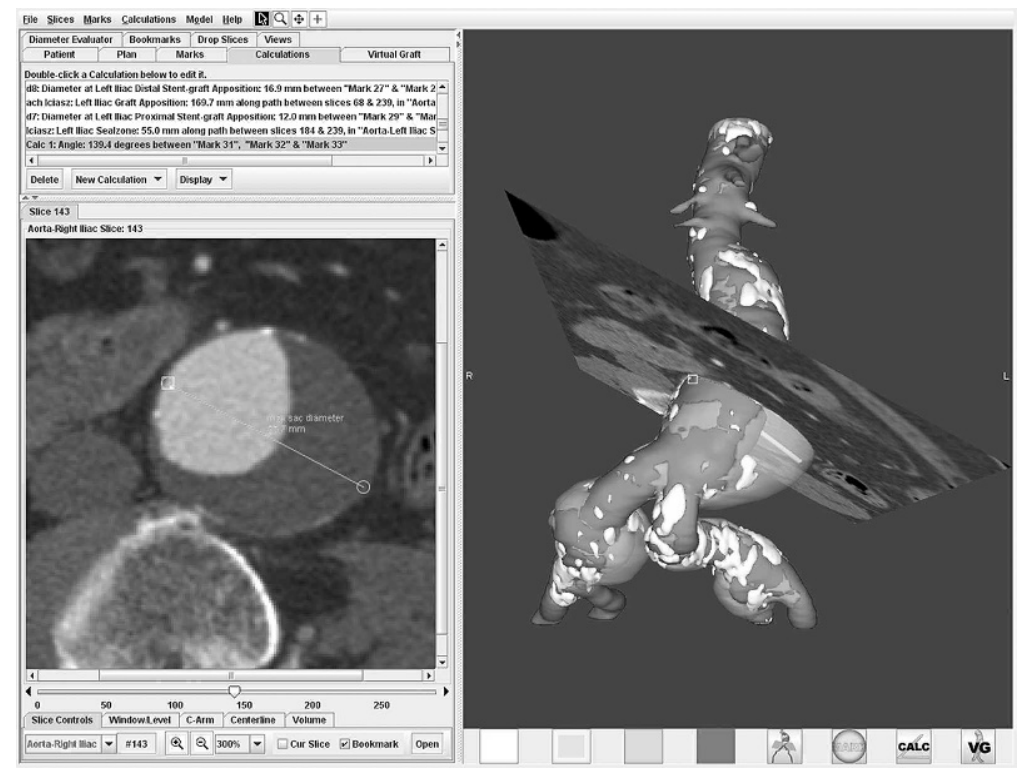

Figure $2 \triangleleft$ The reconstructed 3D models were used to calculate aneurysm neck angulation and identify the orthogonal slice of interest for diameter measurement.

phase, centerlines were generated through the lumen of the vessel. Using this centerline, orthogonal 2D images were created perpendicular to the centerline, thereby showing a true cross section of the vessel. Furthermore, the centerline was used as a measuring tape to calculate blood vessel length, which allowed accurate measuring of diameter, length, and angulation of the blood vessel with a minimum threshold of $1.0 \mathrm{~mm}$. To assess intraobserver variability, 20 datasets were analyzed twice. These datasets were selected at random and blinded for patient data and the results of the initial assessment.

\section{Statistical Analysis}

Dynamic changes (from diastole to systole) in aortic diameter, length, and angulation were analyzed for significance using $t$ tests for paired data; $p<0.05$ was considered to signify a significant difference. Continuous data were reported as the mean \pm standard deviation.

For each measurement, intraobserver variability was assessed using the difference of means analysis described by Bland and Altman. ${ }^{18}$ In this statistical technique, the difference in each pair of measurements was plotted against the pair's mean. The standard devia-

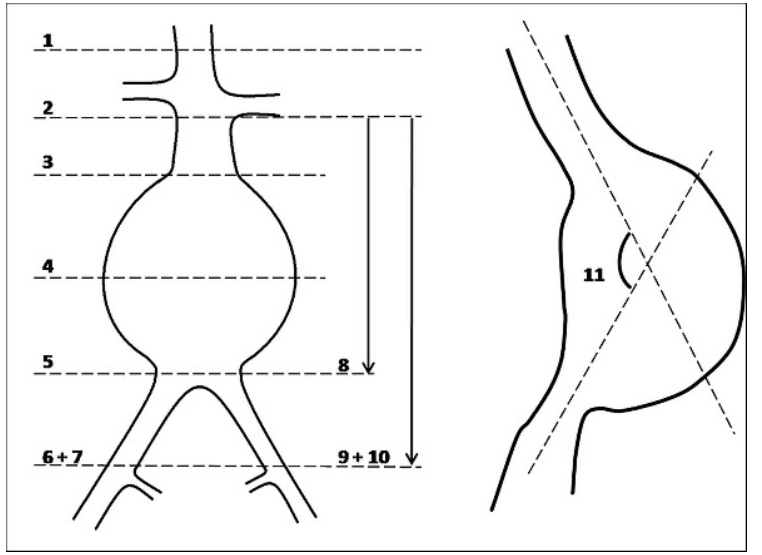

Figure $3 \bullet$ Aortic morphology was assessed based upon 11 different indices of aortoiliac morphology: (1) suprarenal diameter $(5 \mathrm{~mm}$ above the proximal renal artery), (2) proximal neck diameter $1 \mathrm{~mm}$ below the origin of the most distal renal artery, (3) diameter $15 \mathrm{~mm}$ below the distal renal artery, (4) maximal aneurysm diameter, (5) diameter at the aortic bifurcation, (6) diameter at the distal right $\mathrm{CIA}$, (7) diameter at the distal left $\mathrm{CIA},(8)$ infrarenal aortic length (distance between the distal renal artery and aortic bifurcation), (9) aortoiliac length (distance between the distal renal and right hypogastric arteries), (10) aortoiliac length (distance between the distal renal and left hypogastric arteries), and (11) proximal aneurysm neck angulation. 


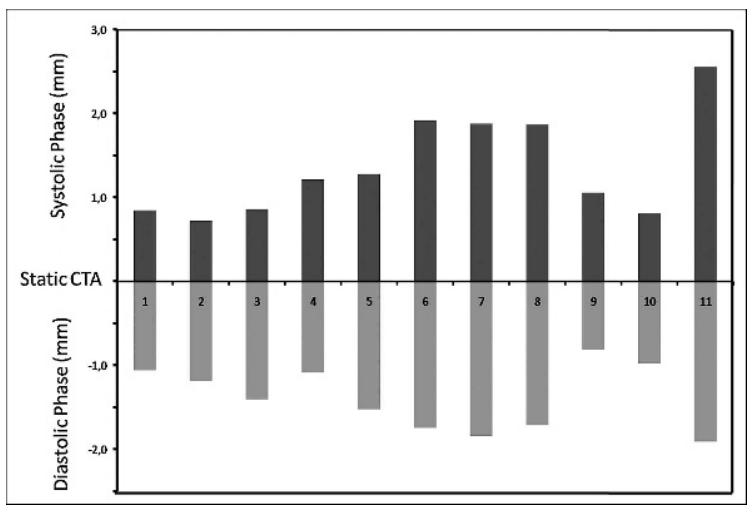

Figure $4 \diamond$ Difference between measurements obtained from static CTA and the systolic and diastolic phase of dynamic CTA. The $x$ axis represents the result from static CTA at the different levels (1: suprarenal diameter, 2: diameter at distal renal artery, 3: diameter $15 \mathrm{~mm}$ below the distal renal artery, 4: maximal diameter, 5: diameter at the bifurcation, 6: right $\mathrm{CIA}$ diameter, 7: left CIA diameter, 8: distance from the distal renal artery to the aortic bifurcation, 9: distance between the distal renal and the right hypogastric arteries, 10: distance between the distal renal and left hypogastric arteries, 11: proximal aneurysm neck angulation); the $y$ axis represents the increase and decrease in diameter, length, and angulation during systole and diastole, respectively.

tion of the mean difference was determined, and the repeatability coefficient (RC) was calculated as 1.96 times the standard deviation. If the observed difference in size between systole and diastole exceeded the RC (with a $95 \%$ confidence interval), the difference was likely not the result of measurement error but probably arose from aneurysm pulsatility.

\section{RESULTS}

Compared to static CTA, mean aneurysm dimensions were smaller at diastole and larger at systole (Fig. 4). At the clinically important proximal and distal endograft sealing zones, aortic and common iliac artery (CIA) diameter changed significantly during the cardiac cycle. Mean aortic diameter at the proximal neck was $21.4 \pm 3.0 \mathrm{~mm}$ at diastole and $23.2 \pm 0.9 \mathrm{~mm}$ at systole, a mean increase of $1.8 \pm 0.4 \mathrm{~mm}$ (8.5\%; $p<0.01)$. Maximal increase in proximal neck diameter was $2.6 \mathrm{~mm}$ (13.7\%; $p<0.01$ ). At a level $15 \mathrm{~mm}$ below the distal renal artery, the mean aortic diameter

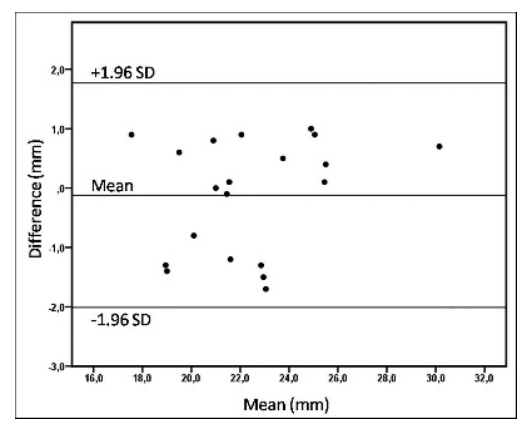

Figure $5 \bullet$ Bland-Altman plot for measurement of aortic diameter at the proximal aneurysm neck. The mean of 2 observations ( $x$ axis) is plotted against the difference between both measurements (y axis). The repeatability coefficient $(R C)$ is defined as 1.96 times the standard deviation of the mean difference.

increase was $2.3 \pm 0.6 \mathrm{~mm}(8.8 \% ; p<0.01)$. At the distal sealing zone, the mean increase in $\mathrm{ClA}$ diameter was $1.7 \pm 0.3 \mathrm{~mm}(14.1 \% ; \mathrm{p}<$ $0.01)$ for the right and $1.8 \pm 0.5 \mathrm{~mm}(14.2 \%$; $\mathrm{p}<0.01)$ for the left $\mathrm{CIA}$. The largest increase in CIA diameter was $2.2 \mathrm{~mm}$ (20.6\%).

As for aneurysm diameter, aneurysm length and proximal aneurysm neck angulation increased from diastole to systole. Mean increase in infrarenal aortic length was $3.7 \pm 0.9 \mathrm{~mm}(3.1 \%, p<0.01)$. The mean increase in right and left aortoiliac lengths were $3.7 \pm 1.6 \mathrm{~mm}(1.9 \%, \mathrm{p}<0.01)$ and $3.6 \pm 1.1 \mathrm{~mm}$ $(1.9 \%, p<0.01)$, respectively. Although the mean increase in the aortoiliac length was small, individual length changes of up to $8.9 \mathrm{~mm}(3.7 \%)$ were observed.

Based upon the Bland-Altman plot (Fig. 5), the RC for the aortic diameter at the level of the proximal aneurysm neck was $1.9 \mathrm{~mm}(8.9 \%)$, approximating the average change in proximal aneurysm neck diameter $(1.8 \pm 0.4 \mathrm{~mm})$. In 13 $(72 \%)$ patients, the observed increase in the proximal aneurysm neck diameter did not exceed the RC (Fig. 6). At both distal sealing zones, however, the mean increase in CIA diameter did exceed the RC (Table).

\section{DISCUSSION}

Changes in aneurysm dimension during the cardiac cycle could result in a mismatch of graft to aneurysm dimensions, potentially leading to type I endoleak and stent-graft migration. $^{9-12,19}$ In the present study, the 


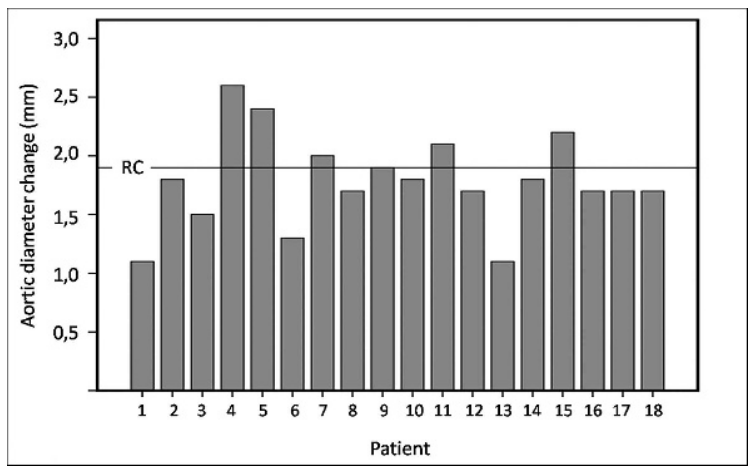

Figure $6 \diamond$ Dynamic changes in proximal aneurysm neck diameter. RC indicates the repeatability coefficient for measurement of aortic diameter at this level. In 5 patients, the observed change in aortic diameter exceeds the $\mathrm{RC}$; in these patients, the observed change in diameter is most likely the result of aortic pulsatility during the cardiac cycle. In the remaining 13 patients, the observed difference between the systolic and diastolic dimensions could merely result from observer variability.

observed mean increase in aortic diameter at the proximal sealing zone was small and in the range of intraobserver variability, so it is more likely to have resulted from intraobserver variability than aneurysm neck pulsatility. However, in a substantial proportion of patients, the increase in proximal aortic diameter exceeded the manufacturer's $10 \%$ stent-graft oversizing recommendation. In contrast to the proximal sealing zone, dynamic changes in the distal sealing zone diameter did exceed intraobserver variability in most patients. Endograft oversizing with a minimum of $10 \%$ based on static CTA could lead to migration and type I endoleak in a substantial proportion of patients. Therefore, oversizing of at least $20 \%$ should be considered.

We used 11 datasets to determine maximum and minimum diameters because it was impossible to select an overall systolic and diastolic dataset. In the first place, the ECG registers the electrical activity of the heart and not contractility. Secondly, the distance between the heart and abdominal aorta results in an unknown delay between ECG registration and a change in abdominal aortic dimensions.

Contrary to previous dynamic imaging studies measuring in only 2 dimensions, ${ }^{9,19}$ we used 3D reconstructions to quantify dynamic changes in aneurysm length and angulation.
TABLE

Change in Diameter, Length, and Angulation Over the Cardiac Cycle and the Corresponding Repeatability Coefficient (RC)

\begin{tabular}{|c|c|c|}
\hline & Change, $\mathrm{mm}$ & $\mathrm{RC}$ \\
\hline \multicolumn{3}{|l|}{ Diameter } \\
\hline Suprarenal & $1.9 \pm 0.5$ & $1.9(8.9 \%)$ \\
\hline Renal & $1.8 \pm 0.4$ & $1.9(8.9 \%)$ \\
\hline $\begin{array}{l}15 \mathrm{~mm} \text { below the } \\
\text { distal renal artery }\end{array}$ & $2.3 \pm 0.6$ & $2.1(7.5 \%)$ \\
\hline Maximal & $2.3 \pm 0.6$ & $2.3(4.1 \%)$ \\
\hline Bifurcation & $2.8 \pm 0.7$ & $2.4(13.8 \%)$ \\
\hline Right CIA & $1.7 \pm 0.3$ & $1.3(10.0 \%)$ \\
\hline Left CIA & $1.8 \pm 0.5$ & $1.6(12.6 \%)$ \\
\hline \multicolumn{3}{|l|}{ Length } \\
\hline Renal-bifurcation & $3.7 \pm 1.0$ & $3.1(2.6 \%)$ \\
\hline Renal-RHA & $3.7 \pm 1.6$ & $3.2(2.0 \%)$ \\
\hline Renal-LHA & $3.6 \pm 1.1$ & $3.4(1.9 \%)$ \\
\hline \multicolumn{3}{|l|}{ Angulation } \\
\hline Proximal neck, ${ }^{\circ}$ & $4.5 \pm 1.5$ & $4.7(3.3 \%)$ \\
\hline
\end{tabular}

This approach has several advantages. First, it allows accurate assessment of infrarenal aortic length, essential to select the appropriate length of the endograft's main body. Because of the small dynamic changes in infrarenal aortic length and the stepwise increase in standard working length for most commercially available endografts, it is, however, unlikely that dynamic CTA would result in the selection of a different main body. In terms of iliac limb length, the mean dynamic increase in aortoiliac length was small, but length changes of up to $8.9 \mathrm{~mm}$ were observed in individual patients. Size changes this large could affect endograft selection. However, it is not always necessary to deploy the iliac limb at the origin of the hypogastric artery. Moreover, the degree of overlap (connection) with the main body and thus final iliac graft length are often determined using additional intraoperative catheter-based length measurements. Although there is some kinking of the aneurysm and aorta during the cardiac cycle, changes in proximal aortic neck angulation are small and do not affect endograft selection.

Secondly, changes in aneurysm morphology are not restricted to $2 \mathrm{D}$ changes in diameter or circumference. ${ }^{9}$ When using 2D 
cross-sectional imaging only, movement of the aneurysm in the cranial or caudal (out of plane) direction could result in comparing aortic diameter at different sites (above or below the original slice) instead of different phases of the cardiac cycle. By using 3D aneurysm models, we were able to identify the site of interest for every phase of the cardiac cycle, irrespective of cranial to caudal movement, kinking, or out of plane translation of 2D cross-sectional images.

\section{Conclusion}

The observed changes in aneurysm dimension during the cardiac cycle are small and in the range of intraobserver variability. Although dynamic changes in aneurysm length and proximal aneurysm neck diameter have little impact on preoperative endograft selection, oversizing of up to $20 \%$ should be considered to prevent distal type I endoleak.

\section{REFERENCES}

1. Brewster DC, Cronenwett JL, Hallett JW, et al. Guidelines for the treatment of abdominal aortic aneurysms. Report of a subcommittee of the Joint Council of the American Association for Vascular Surgery and Society for Vascular Surgery. J Vasc Surg. 2003;37:1106-1117.

2. Chaikof EL, Fillinger MF, Matsumura JS, et al. Identifying and grading factors that modify the outcome of endovascular aortic aneurysm repair. J Vasc Surg. 2002;35:1061-1066.

3. Waasdorp EJ, Vries JP, Hobo R, et al. Aneurysm diameter and proximal aortic neck diameter influence clinical outcome of endovascular abdominal aortic repair: a 4-year EUROSTAR experience. Ann Vasc Surg. 2005;19:755-761.

4. Beebe HG, Kritpracha B, Serres $S$, et al. Endograft planning without preoperative arteriography: a clinical feasibility study. J Endovasc Ther. 2000;7:8-15.

5. Parker MV, O'Donnell SD, Chang AS, et al. What imaging studies are necessary for abdominal aortic endograft sizing? A prospective blinded study using conventional computed tomography, aortography, and three-dimensional computed tomography. J Vasc Surg. 2005;41:199-205.

6. Broeders IA, Blankensteijn JD. Preoperative imaging of the aortoiliac anatomy in endovascular aneurysm surgery. Semin Vasc Surg. 1999;12:306-314.
7. Fillinger MF. New imaging techniques in endovascular surgery. Surg Clin North Am. 1999;79:451-475.

8. Wyers MC, Fillinger MG, Schermerhorn ML, et al. Endovascular repair of abdominal aortic aneurysm without preoperative arteriography. J Vasc Surg. 2003;38:730-738.

9. Teutelink A, Rutten A, Muhs BE, et al. Pilot study of dynamic cine CT angiography for the evaluation of abdominal aortic aneurysms: implications for endograft treatment. $J$ Endovasc Ther. 2006;13:139-144.

10. Teutelink A, Muhs BE, Vincken KL, et al. Use of dynamic computed tomography to evaluate pre-and postoperative aortic changes in AAA patients undergoing endovascular aneurysm repair. J Endovasc Ther. 2007;14:44-49.

11. Laskowski I, Verhagen HJ, Gagne PJ, et al. Current state of dynamic imaging in endovascular aortic aneurysm repair. J Endovasc Ther. 2007;14:807-812.

12. Truijers $M$, Fillinger MF, Renema KW, et al. Invivo imaging of changes in abdominal aortic aneurysm thrombus volume during the cardiac cycle. J Endovasc Ther. 2009;16:314-319.

13. Aziz I, Lee J, Lee JT, et al. Accuracy of threedimensional simulation in the sizing of aortic endoluminal devices. Ann Vasc Surg. 2003;17: 129-136.

14. Beebe HG, Kritpracha B, Serres S, et al. Endograft planning without preoperative arteriography: a clinical feasibility study. J Endovasc Ther. 2000;7:8-15.

15. Beebe HG, Kritpracha B. Computed tomography scanning for endograft planning: evolving toward three-dimensional, single source imaging. Semin Vasc Surg. 2004;17:126-134.

16. Ouriel K, Tanquilut E, Greenberg RK, et al. Aortoiliac morphologic correlations in aneurysms undergoing endovascular repair. J Vasc Surg. 2003;38:323-328.

17. Sprouse LR, Meier GH, Parent FN, et al. Is threedimensional computed tomography reconstruction justified before endovascular aortic aneurysm repair? J Vasc Surg. 2004;40:443-447.

18. Bland JM, Altman DG. Statistical methods for assessing agreement between two methods of clinical measurement. Lancet. 1986;1(8476): 307-310.

19. van Herwaarden JA, Bartels LW, Muhs BE, et al. Dynamic magnetic resonance angiography of the aneurysm neck: conformational changes during the cardiac cycle with possible consequences for endograft sizing and future design. J Vasc Surg. 2006;44:22-28. 